\title{
Lab-on-chips based testing methods to investigate the mechanical behaviour of thin films
}

\author{
P. Carbonnelle ${ }^{1,2}$, S. Ryelandt ${ }^{3}$, A. Boé, ${ }^{2,3}$, M. Coulombier ${ }^{3}$, A. Zulfiqar², U. Bhaskar², J.-P. Raskin ${ }^{2}$ \\ and T. Pardoen ${ }^{3}$ \\ 1 MEMS Instruments, 1348 Louvain-la-Neuve, Belgium \\ 2 Electrical Engineering, Université catholique de Louvain, 1348 Louvain-la-Neuve, Belgium \\ 3 Institute of Mechanics, Materials and Civil engineering, Université catholique de Louvain, 1348 \\ Louvain-la-Neuve, Belgium
}

The characterization of the elastic, plastic and fracture behavior of nanofilms is extremely challenging, from the preparation and manipulation of specimens to the application of small loads and extraction of accurate stresses and strains. Several novel lab-on-a-chips concepts will be presented to address these challenges. The first relies on the internal stress present in one film to provide the actuation for deforming the sample film [1]. The second lab-on-a-chip relies on a macroscopic, external actuation on the silicon chip.

Classical MEMS-based microfabrication procedures are used to pattern the films into special test structures and to release them from the substrate. The actuation results in uniform strain, so that data analysis is straightforward. The measurement of the displacement gives access to the stress and the strain applied to the sample, provided that the Young's modulus and mismatch strain of the films are known. A third lab-on-chip performs the measurement of Young's

The characterization of the elastic, plastic and modulus when needed. Various fracture behavior of nanofilms is extremely combinations of dimensions allow the challenging, from the preparation and characterization of different materials and manipulation of specimens to the application mechanical properties. Results obtained on of small loads and extraction of accurate both ductile and brittle nanofilms demonstrate stresses and strains. Several novel lab-on-a-the interest of these methods. chips concepts will be presented to address these challenges. The first relies on the internal stress present in one film to provide the actuation for deforming the sample film [1]. The second labon-a-chip relies on a macroscopic, external actuation on the silicon chip. modulus when needed. Various combinations of dimensions allow the characterization of different materials and mechanical properties. Results obtained on both ductile and brittle nanofilms demonstrate the interest of these methods.

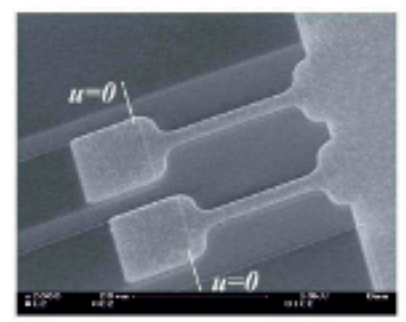

Fig. 1. Tensile test structures before release showing the dog bone shape of specimens.

1. Gravier et al, Journal of MEMS, 18, 3, June 2009

This is an Open Access article distributed under the terms of the Creative Commons Attribution-Noncommercial License 3.0, which permits unrestricted use, distribution, and reproduction in any noncommercial medium, provided the original work is properly cited. 